\title{
Spontaneous Epidural Hematoma Associated with Venous Phlebolith in Cervical Spine and Possible Pathogenesis
}

\section{Dong Hwan Kim, Dong Ha Kim, Kyoung Hyup Nam, Byung Kwan Choi, In Ho Han}

Department of Neurosurgery \& Medical Research Institute, Pusan National University Hospital, Pusan National University School of Medicine, Busan, Korea

\section{Corresponding Author:}

In Ho Han

Department of Neurosurgery \& Medical Research Institute, Pusan National University Hospital, 179 Gudeok-ro, Seo-gu, Busan 49241, Korea

Tel: +82-51-240-7257

Fax: +82-51-244-0282

E-mail: farlateral@hanmail.net

Received: February 10, 2017

Revised: August 18, 2017

Accepted: August 21, 2017

Copyright ( $~ 2017$ by The Korean Spinal Neurosurgery Society

This is an open access article distributed under the terms of the Creative Commons Attribution Non-Commercial License (http://creativecommons.org/licenses/bync/4.0/) which permits unrestricted noncommercial use, distribution, and reproduction in any medium, provided the original work is properly cited.

Although the etiology of spontaneous spinal epidural hematoma (SSEH) is unclear, SSEH is known to be associated with anticoagulants, coagulopathy, vascular malformation, hypertension, and pregnancy. However, no report has been issued on the relation between SSEH and venous phlebolith. Here, the authors present an extremely rare case of SSEH associated with phlebolith in the cervical spine and suggest a possible pathogenesis. A 36-year-old man without any relevant medical history presented with neck pain and numbness and severe radiating pain on the left arm. Magnetic resonance imaging showed epidural hematoma at the C5-7 level, and computed tomography revealed a calcified nodule in the left epidural hemorrhage at $\mathrm{C} 6$ level. During left partial laminectomy, epidural venous plexus, and thick epidural hematoma were found, and hematoma removal revealed a white, ovoid, smooth, hard mass of diameter $3 \mathrm{~mm}$. Histopathologic examination confirmed the mass as a venous phlebolith. The presence of a calcified solitary nodule in dorsal epidural space indicates the presence of phlebolith and the risk of SSEH. In such cases, the authors recommend spine surgeons should take into consideration the possibility of epidural hemorrhage.

Key Words: Spinal epidural hematoma, Phlebolith, Venous malformation

\section{INTRODUCTION}

Spontaneous spinal epidural hematoma (SSEH) is an uncommon disease that causes sudden spinal cord compression and neurological deterioration ${ }^{11)}$. Its annual incidence is about 0.1 per 100,000 persons, though this has increased since the advent of computed tomography (CT) and magnetic resonance imaging (MRI). SSEH may affect every age, but especially those in the fourth or fifth decade ${ }^{7,12)}$. Its etiology is unclear, but is known to be associated with anticoagulants, coagulopathy, vascular malformation, and hypertension ${ }^{4,7,2)}$. However, in $40 \%$ of reported patients, no definite predisposing factor was found ${ }^{12}$.

A phlebolith is small, local, usually round calcification found within a vein. It is most frequently encountered in a pelvic vein and generally is of no clinical importance ${ }^{8)}$. However, the next most common site is the head and neck, where it is associated with hemangioma or venous malformation ${ }^{6}$. On the other hand, the presence of a phlebolith in the cervical epidural space has never been previously reported. Here, we report SSEH associated with a venous phlebolith, which suggests a possible pathogenesis, and provide a review of the literature.

\section{CASE REPORT}

A 36-year-old man with no relevant medical history presented with neck pain and numbness and severe radiating pain in the left arm, which developed suddenly during sleep. Neurological examination revealed severe radiating pain on the left C7 and C8 dermatomes. Motor functions were normal.

There were no abnormal findings on cervical plain radiography (Fig. 1). CT demonstrated a space-occupying lesion of high density in the dorsal epidural space from $\mathrm{C} 6$ to $\mathrm{C} 7$, and interestingly, depicted a calcified nodule located in the epidural hematoma at the C6 level (Fig. 2). MRI demonstrated epidural hematoma of high intensity on T2-weighted images and of intermediate intensity on T1-weighted images at the C6 to C7 level, containing a nodular, low intensity focus corresponding to calcification (Fig. 3). No other abnormal vascular structures were identified by cervical MRI.

We decided to perform an emergency operation. And written informed consent was obtained from patient. After left partial laminectomy to C6/7 level, the epidural venous plexus and thick epidural hematoma were found, and on removing the hematoma, a white, ovoid, smooth, 


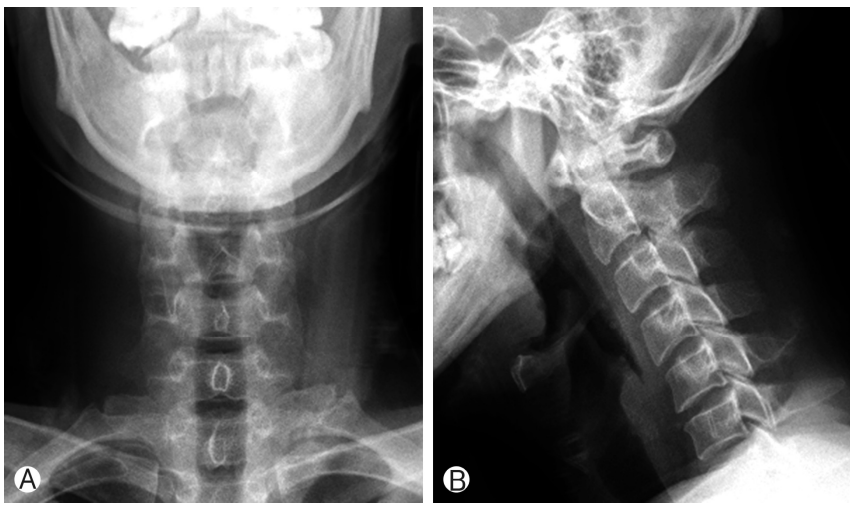

Fig. 1. (A, B) There were no abnormal findings on cervical plain radiography.

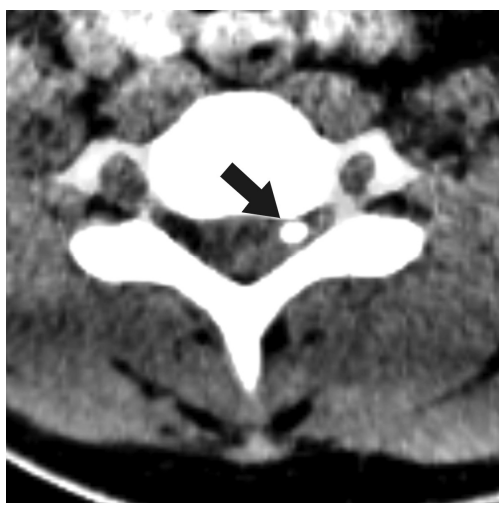

Fig. 2. Computed tomography shows small calcified nodule (black arrow) at C6 level.

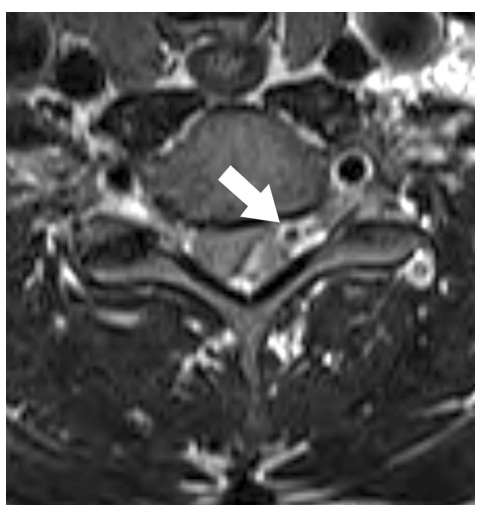

Fig. 3. Axial T2-weighted image in magnetic resonance imaging shows high signal intensity containing nodular hypointense focus (white arrow).

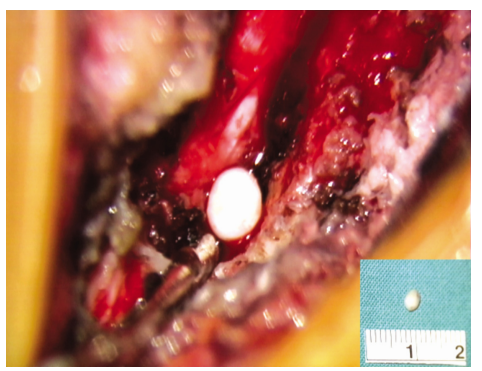

Fig. 4. A phlebolith with 3-mm diameter was observed in intraoperative field.

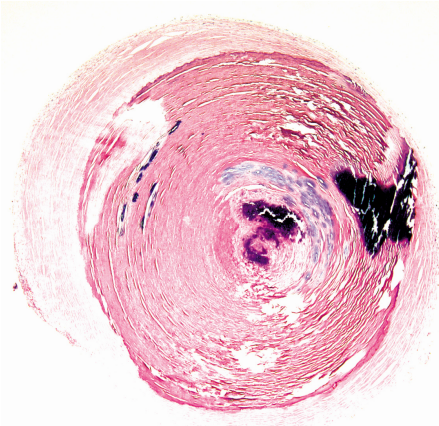

Fig. 5. Histopathologic finding of the phlebolith. Calcium deposit and aggregation of collagen fibers are identified. They form round and stone like structures $(\mathrm{H} \& \mathrm{E}$, $\times 40)$.

hard mass of diameter $3 \mathrm{~mm}$ was encountered during manipulation of the venous plexus (Fig. 4). Postoperatively, the radiating pain was completely relieved without neurologic deficits. Histopathologic examination confirmed a phlebolith, consisting of a calcified thrombus with a concentric pattern of mineralization, surrounded by granulation tissue and small capillaries under a hyaline layer (Fig. 5).

\section{DISCUSSION}

Anticoagulant administration or coagulation disorders are the most common causes of SSEH, and these are followed by vascular malformation ${ }^{3)}$. However, in $40 \%$ of cases, no definite predisposing factor is identified ${ }^{12)}$. In these idiopathic cases, SSEH was believed to originate from a venous plexus. Fragile, valveless, epidural venous plexuses connect to the abdominal and thoracic venous systems, and increased pressure in the intra-abdominal or intrathoracic venous systems induced by sneezing, coughing, voiding or performing some other Valsalva maneuver might elevate intraspinal venous plexus pressure sufficiently to cause epidural venous plexus rupture and hematoma formation".

In the present case, SSEH was suspected to originate from the epidural venous plexus based on intraoperative findings, and the epidural hematoma was presumed to be associated with the phlebolith found in the epidural venous plexus.

Spinal epidural phlebolith has not been previously reported, and thus, is unfamiliar to spine surgeons. Phleboliths are produced by calcification of old thrombi in veins, and are most frequently found in pelvic veins, which are subject to stress injury during defecation $^{8)}$. In fact, approximately $40 \%$ of the normal population have a phlebolith as determined by pelvic radiography ${ }^{10)}$. The next most common site is the head and neck area, where they are found in $15 \%$ to $25 \%$ of intramuscular hemangiomas ${ }^{1,8)}$.

The pathogenesis of phlebolith is believed to be associated with thrombus formation resulting from vessel wall intimal injury or blood flow stagnation ${ }^{2}$. The thrombus formed then calcifies to form the phlebolith core and the surrounding fibrinous component undergoes secondary calcification and attaches to this core $^{\text {6) }}$. Repetition of these processes cause phlebolith growth and produces a characteristic concentric ring or 'onion-like' appearance $^{5)}$. 
Basis on proposed theories, we speculate slow blood flow in the cervical epidural venous plexus caused a thrombus in our patient, and that repeated calcification and fibrous adhesion resulting in phlebolith formation, which in turn caused vein wall erosion or provoked local coagulopathy and finally caused epidural hemorrhage. It should also be noted a preexisting venous malformation or cavernous angioma can cause thrombus and phlebolith formation followed by hemorrhage. Although we were not able to identify abnormal vascular structures, there was a possibility of a preexisting venous malformation in the epidural space in our patient.

The diagnosis of phlebolith in cervical plain radiographs can be difficult because they are generally only $1.5-4 \mathrm{~mm}$ in diameter and are likely to be obscured by spinal bony structures in this location. Thus, CT is important for the diagnosis of epidural phlebolith. In the described case, axial CT visualized the phlebolith as a small round radiopaque mass located in the lateral dorsal epidural space near the intervertebral foramen.

\section{CONCLUSION}

We report the first case of SSEH associated with phlebolith on the cervical spine, which presented as neurologic deficit in 36-year-old man. After total surgical removal of SSEH and phlebolith, the patient fully recovered. We suggest a calcified solitary nodule in the dorsal epidural space be considered pathognomic for phlebolith and the risk of SSEH, and that in such cases, spine surgeons carefully consider the possibility of epidural hemorrhage.

\section{CONFLICT OF INTEREST}

No potential conflict of interest relevant to this article was reported.

\section{REFERENCES}

1. Alami B, Lamrani Y, Addou O, Boubbou M, Kamaoui I, Maaroufi $\mathrm{M}$, et al: Presumptive intramuscular hemangioma of the masseter muscle. Am J Case Rep 16:16-19, 2015

2. Choi HJ, Lee JC, Kim JH, Lee YM, Lee HJ: Cavernous hemangioma with large phlebolith of the parotid gland. J Craniofac Surg 24: e621-623, 2013

3. Dziedzic T, Kunert P, Krych P, Marchel A: Management and neurological outcome of spontaneous spinal epidural hematoma. J Clin Neurosci 22:726-729, 2015

4. Groen RJ, Ponssen H: The spontaneous spinal epidural hematoma. A study of the etiology. J Neurol Sci 98:121-138, 1990

5. Hassani A, Saadat S, Moshiri R, Shahmirzadi S: Hemangioma of the buccal fat pad. Contemp Clin Dent 5:243-246, 2014

6. Kato H, Ota Y, Sasaki M, Arai T, Sekido Y, Tsukinoki K: A phlebolith in the anterior portion of the masseter muscle. Tokai J Exp Clin Med 37:25-29, 2012

7. Lee HH, Park SC, Kim Y, Ha YS: Spontaneous spinal epidural hematoma on the ventral portion of whole spinal canal: a case report. Korean J Spine 12:173-176, 2015

8. Mandel L, Perrino MA: Phleboliths and the vascular maxillofacial lesion. J Oral Maxillofac Surg 68:1973-1976, 2010

9. Paraskevopoulos D, Magras I, Polyzoidis K: Spontaneous spinal epidural hematoma secondary to extradural arteriovenous malformation in a child: a case-based update. Childs Nerv Syst 29:19851991, 2013

10. Shemilt P: The origin of phleboliths. Br J Surg 59:695-700, 1972

11. Yamao Y, Takagi Y, Kawauchi T, Arakawa Y, Takayama M, Miyamoto S: Surgical management of recurrent spontaneous spinal epidural hematoma with 3 episodes. Spine (Phila Pa 1976) 40:E996998, 2015

12. Zhong W, Chen H, You C, Li J, Liu Y, Huang S: Spontaneous spinal epidural hematoma. J Clin Neurosci 18:1490-1494, 2011 